\title{
The distribution of important sero-complexes of flaviviruses in Malaysia
}

\begin{abstract}
Flaviviruses (FVs) are arthropod-borne viruses of medical and veterinary importance. Numerous species of FVs have been isolated from various host; mainly humans, animals, ticks, and mosquitoes. Certain FVs are extremely host-specific; at the same time, some FVs can infect an extensive range of species. Based on published literatures, 11 species of FVs have been detected from diverse host species in Malaysia. In humans, dengue virus and Japanese encephalitis virus have been reported since 1901 and 1942. In animals, the Batu Cave virus, Sitiawan virus, Carey Island, Tembusu virus, Duck Tembusu virus, and Japanese encephalitis viruses were isolated from various species. In mosquitoes, Japanese encephalitis virus and Kunjin virus were isolated from Culex spp., while Zika virus and Jugra virus were isolated from Aedes spp. In ticks, the Langat virus was isolated from Ixodes spp. One of the major challenges in the diagnosis of FVs is the presence of sero-complexes as a result of crossreactivity with one or more FV species. Subsequently, the distribution of specific FVs among humans and animals in a specific population is problematic to assess and often require comprehensive and thorough analyses. Molecular assays such as quantitative reverse transcriptase polymerase chain reaction (qRT-PCR) and digital droplet RT-PCR (ddRT-PCR) have been used for the differentiation of flavivirus infections to increase the accuracy of epidemiological data for disease surveillance, monitoring, and control. In situations where sero-complexes are common in FVs, even sensitive assays such as qRT-pCR can produce false positive results. In this write up, an overview of the various FV sero-complexes reported in Malaysia to date and the challenges faced in diagnosis of FV infections are presented.
\end{abstract}

Keyword: Animal; Arthropod; Flavivirus; Human; Sero-complex 